\title{
METODOLOGIAS ATIVAS DE APRENDIZAGEM COMO PRÁTICA COLABORATIVA: MESCLANDO SALA DE AULA INVERTIDA E RPG NO CURSO DE ENGENHARIA DE PRODUÇÃO
}

Silvana Rodrigues Quintilhano-squintilhano@utfpr.edu.br

Universidade Tecnológica Federal do Paraná - Campus Londrina

Departamento de Engenharia de Produção

Estrada dos Pioneiros - Jardim Morumbi

86036-370 - Londrina - PR

Rogério Tondato-rogeriotondato@utfpr.edu.br

Universidade Tecnológica Federal do Paraná -Campus Londrina

Departamento de Engenharia de Produção

Estrada dos Pioneiros - Jardim Morumbi

86036-370 - Londrina - PR

Felipe Guilherme Oliveira-Melo-felipe.guilherme@univasf.edu.br Universidade Federal do Vale do São Francisco, Campus Salgueiro Rua Antônio Figueira Sampaio, 134 - Nossa Sra. das Graças 56000-000 - Salgueiro - PE

Ana Carolina Piccinini de Alencar Schiavi-anaschiavi@alunos.utfpr.edu.br Universidade Tecnológica Federal do Paraná, Campus Londrina

IC/ Engenharia de Produção

Estrada dos Pioneiros - Jardim Morumbi

86036-370 - Londrina - PR

Resumo: O cenário educacional traz uma longa trajetória de ensino pautado na transmissão de conhecimento unilateral, equivocadamente centrada no professor, e o aluno figurava como mero agente passivo no processo de aprendizagem, acarretando um alto índice de evasão e reprovação. Na busca por atualização docente, surge as metodologias ativas, que buscam direcionar as estratégias de ensino para uma participação ativa e crítica do aluno, estimulando raciocínio lógico e uma relação eficiente entre teoria e prática, aliadas aos suportes tecnológicos. Sob este escopo, o objetivo desse artigo será analisar a aplicação de metodologias ativas, mesclando Sala de Aula Invertida e Role Play Game, na disciplina de Comportamento Humano do curso de Engenharia de Produção da UTFPR-Campus Londrina, no intuito evidenciarmos a eficiência dos métodos, bem como examinar a viabilidade de integração de várias metodologias na abordagem de um conteúdo. Para tanto, utilizou-se o método da pesquisa-ação, de caráter exploratório. Os resultados dessa pesquisa possibilitaram concluir que esses modelos permitem interação e motivação dos alunos em sala de aula, atingindo $100 \%$ de satisfação na aquisição de conhecimento, bem como $81 \%$ de satisfação dos alunos através da participação e envolvimento, ou seja, ao mesclar duas metodologias minimizou as lacunas de aprendizagem previstas pela heterogeneidade da sala de aula.

Palavras-chave: Metodologia Ativa. Sala de Aula Invertida. Role Play Game. Engenharia de Produção. 


\section{INTRODUÇÃo}

Com o surgimento da era digital e das inovações tecnológicas, o ensino tradicional tornouse ineficiente no processo de ensino-aprendizagem, pois a forma de conhecer tornou-se múltipla, variada e, excepcionalmente, acessível. Porém, ainda é muito recorrente aulas expositivas, reforçando os altos índices de reprovação e desistências, principalmente no Ensino Superior.

Repensar o contexto de sala aula tornou-se uma preocupação no cenário educacional. Nesta perspectiva, aliadas às novas Tecnologias de Informação e Comunicação, surgem as Metodologias Ativas de Aprendizagem, inovando o modo de ensinar. Tais métodos permitem uma maior interação do aluno em sala de aula, enquanto o professor atua como mediador no processo de ensino-aprendizagem.

Nessa pesquisa, tratar-se-á da mescla de dois métodos: Sala de Aula Invertida e Role Play Game (RPG - Jogo de Representações), fundamentando os princípios didáticos das Metodologias Ativas, onde o aluno é sujeito ativo no processo de aquisição do conhecimento, enquanto o professor atua como mediador, potencializando a aprendizagem cognitiva.

O objetivo será analisar a aplicação da Sala de Aula Invertida e do RPG, na disciplina de Comportamento Humano do curso de Engenharia de Produção da UTFPR- Campus Londrina, no intuito de evidenciar a eficiência dos métodos, bem como validar a possibilidade de combinar técnicas didáticas, numa simbiose profícua.

Para tanto, será desenvolvido as seguintes etapas: primeira etapa será a apresentação teórica sobre Metodologias ativas, Sala de Aula Invertida e Role Play Game; em seguida será explanado sobre os aspectos metodológicos; e, por fim, a descrição das etapas e aplicação da metodologia, evidenciando os resultados obtidos.

\section{METODOLOGIAS ATIVAS DE APRENDIZAGEM}

Conforme ressalva Eckert, "os novos processos de ensino e aprendizagem da escola moderna começam a enxergar o estudante como indivíduo único, a entender suas particularidades e a compreender suas habilidades e predisposições às disciplinas." (2017, p. 104). Dessa forma, surgem as metodologias ativas de aprendizagem, permitindo aos alunos um maior conhecimento aplicado à realidade. O método de ensino de metodologias ativas tem como principal objetivo a inserção do aluno como agente principal de seu aprendizado. Nessa prática, o aluno interage, tornando-se agente ativo do processo, enquanto o professor assume papel de orientador/mediador.

Barbosa et al. (2018) explicam que as metodologias ativas são utilizadas pelo docente como forma de dar autonomia ao aluno no ato de construir seu conhecimento. Para o sucesso da prática, é possível a utilização de ferramentas tecnológicas como jogos, redes sociais, vídeos e pesquisas, prendendo, dessa forma, ainda mais o interesse do aluno na atividade. Elas podem ser aplicadas a qualquer faixa etária e nível de escolaridade. As metodologias ativas permitem aulas com resoluções de problemas e inserem o aluno tornando-o o principal agente no processo de aprendizagem, desenvolvendo, também, sua habilidade em trabalho em grupo. "No tocante ao ensino, a promoção da mudança deve se ancorar na possibilidade de produzir um processo didático que vá além da transmissão de informação.” (DE PAULA et al., 2018, p. 27)

Santos (2013) citado por Barbosa et al. (2018) menciona que são necessárias quatro condições básicas para a aprendizagem significativa ocorrer: motivação, interesse, habilidade de compartilhar experiências e habilidade de interagir com diferentes contextos. Nesse sentido, a motivação vem para impulsionar a busca pelo conhecimento, o interesse conduz ao foco, o 
compartilhamento de experiências leva a novas atividades e múltiplos conhecimentos e a interação em contextos variados auxilia na construção de habilidades e competências. $\mathrm{O}$ docente deve utilizar o conhecimento do discente para que ele se sinta responsável por seu aprendizado e busque sempre a melhoria deste.

Alguns exemplos de metodologias ativas são: Aprendizado por Pares, Aprendizagem baseada em Projetos, Problem Based Learning (PBL - Aprendizagem baseada em Problemas), Aprendizagem por Times, Estudo de Caso, Pensamento Compartilhado em Pares, Sala de Aula Invertida, Grupo de verbalização e observação - GV-GO, Role-play, Storytelling, entre outras. (AHLERT et. al, 2018; LEAL, MIRANDA; CASA NOVA, 2019)

\subsection{Sala de Aula Invertida}

Conforme Jhonson (2012) apud Rodrigues et al. (2015), na sala de aula invertida, o aluno passa a não possuir mais o papel de observador, porém é necessária a colaboração por parte dele para que as atividades sejam feitas no prazo estabelecido pelo professor. O professor é um mentor e facilitador nesse processo, envolvendo os alunos em discussões, questionando-os e ouvindo suas ideias. Matos (2018) salienta que "inverter a sala de aula significa mudar a mentalidade e deslocar a atenção do professor para o aluno, para a aprendizagem. O professor assume um novo papel, deixa de ser um simples transmissor de informações e passa a ser um curador, um orientador". Esse modelo, segundo Yoshizawa et al. (2017), tem como objetivo otimizar o tempo em sala de aula, possibilitando ao professor verificar oportunidades de aprendizagem e personalizar o processo.

Matos (2018) apresenta o modelo de sala de aula invertida em quatro etapas, conforme a Figura 1.

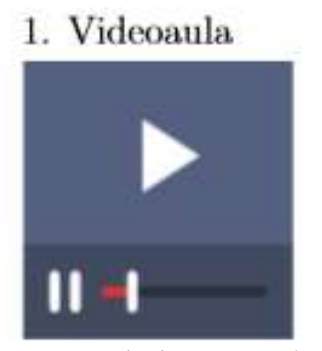

Figura 1 - Etapas da sala de aula invertida.

Fonte: Flaticon apud Matos (2018)
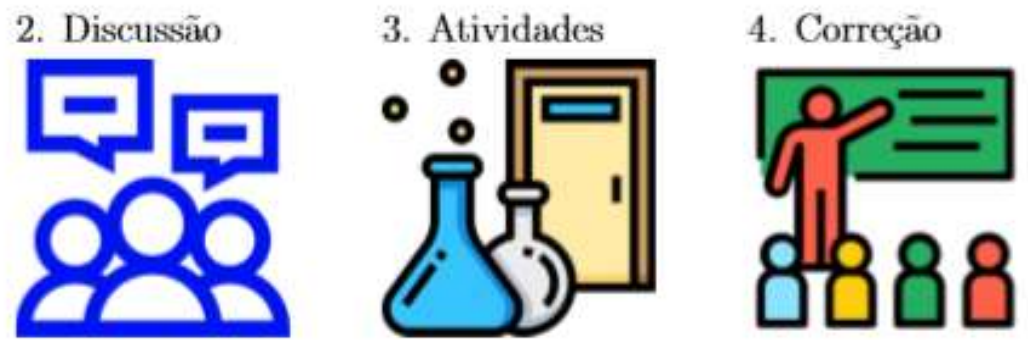

Segundo o autor (MATOS, 2018), na fase de videoaula, busca-se disponibilizar conteúdos aos alunos, podendo estes serem autorais pelo professor ou não, mas necessariamente sendo algo diferenciado e que prenda a atenção do aluno. Todo o tempo investido por ele e pelo professor nessa etapa é tempo útil em sala de aula. A fase de discussão serve para que o professor veja se os alunos realmente assistiram ao vídeo e para a realização de um tira dúvidas sobre o conteúdo tratado. A terceira fase é a maior parte da aula. O tempo de aula que antes servia para a exposição de conteúdos agora serve para a realização de atividades. Para isso, espaço em sala de aula precisa ser repensado, para que haja uma maior participação dos alunos. Essas atividades devem ser acessíveis a todos e com vários níveis de dificuldades para que haja uma evolução do aluno. Por fim, o acompanhamento e correção das atividades mesmo que posta como última etapa, se deve ao feedback que deve ser dado de forma contínua ao longo da aula. Essa tarefa não se deve unicamente ao professor, uma vez que os alunos, enquanto resolvem as atividades, podem se deparar com dificuldades e, sozinhos, solucioná-las. Nessa fase o professor os acompanha, principalmente aqueles que possuem mais dificuldade. 


\subsection{Role Play Game (RPG)}

Segundo Cavalcanti (2009), na educação os jogos vêm sendo há algum tempo utilizados para o auxílio na construção educacional, uma vez que desenvolve diversas características no indivíduo. O RPG está entrando nesse cenário como forma de trazer o lúdico para o aprendizado, para que os alunos encontrem prazer e diversão nas aulas.

Segundo Rezende e Coelho (2013), o RPG foi inventado nos Estados Unidos, tendo como seu precursor o jogo Dungeons \& Dragons e pode ser definido como um "Jogo de Interpretação de Papeis". O RPG surgiu no Brasil no fim da década de 80 por meio de intercambistas vindos dos EUA que trouxeram consigo um material de jogo, que foi disseminado ao país for meio de fotocópias (SCHMIT, 2008).

O RPG proporciona resoluções de situação problema, aplicações de prática no dia a dia, expressão oral, expressão corporal e preocupação e respeito ao outro. "Nesse sentido, ao jogar ou brincar, a criança une o prazer do jogo com a subordinação às regras, que é um elemento importante, pois, ao renunciar ações impulsivas, constrói-se um caminho prazeroso e desafiante no ato de jogar." (REZENDE, COELHO, 2013, p. 2)

Os jogos permitem, além da aprendizagem, o desenvolvimento da coordenação, da atenção, percepção, memorização, inteligência e expressão corporal. No contexto escolar, os jogos devem ser usados no momento certo, conforme a curiosidade e interesse dos alunos, aliado com a proposta do professor.

Cavalcanti (2009) explica que o RPG, ou jogo de interpretação, é um jogo em que um mestre (interpretado por um jogador) conduz todo o cenário e acontecimentos em que os personagens de cada jogador passarão. Ele possui um processo narrativo que vai evoluindo ao longo do decorrer do jogo. Nele o jogador é um participante ativo que escolhe os caminhos por onde trilhar e toma decisões sempre previstas pelo mestre. Algumas características desenvolvidas com o uso do RPG são: as expressões orais e corporais, o incentivo à leitura e determinação de atitudes, ações em grupo e o conteúdo disciplinar e interdisciplinar. (CAVALCANTI, 2009)

Quirino (2013) salienta que jogos com caráter didático têm a função de resolução de situações-problemas, de integração e desenvolvimento da imaginação, além de ser motivacional para o aprendizado. O RPG é um tipo de jogo que o professor consegue juntar a turma toda, não deixando de perceber a individualidade de cada um. De acordo com o autor, "se utilizados de acordo com a proposta pedagógica do professor e da escola, certamente os jogos quebrarão a rigidez do ensino e se tornarão mais uma ferramenta pedagógica." (QUIRINO, 2013, p. 45)

O jogo oferece grande potencial cognitivo ao indivíduo. Oliveira (1995) apud Cabalero (2007) aduz que o desenvolvimento proximal só é possível de se tornar real quando indivíduos de diferentes níveis de conhecimento interagem entre si, em que o mais experiente auxilia o menos experiente. Jogos que envolvem uma situação social e imaginária dependem da experiência anterior do sujeito, ou seja, quanto mais experiência ele tiver, mais sua imaginação irá fluir no jogo. O RPG, com suas regras, pode ser um mediador entre o homem com o mundo e com o outro social.

O que caracteriza essa situação é a grande possibilidade de intercâmbios entre os sujeitos do grupo. Os educandos se indagam mutuamente, e este processo gera, potencialmente, conflitos, que podem ser evidenciados na interação social. No momento em que se encontram envolvidos na atividade de produção escrita em colaboração, os sujeitos trocam conhecimentos, inclusive entre os que trabalham com hipóteses mais avançadas, ou seja, esta construção ocorre na mediação das “zonas de desenvolvimento proximal." (CABALERO, 2007, p. 9) 
Bolzan (2003) apud Cabalero (2007) relata por meio de suas pesquisas que a metodologia RPG motivou os estudantes a potencializar os inter-relacionamentos, o que provou a possibilidade do RPG ser utilizado como auxílio para o aprendizado de comunidades, tanto no presencial como a distância.

Schmit (2008) defende que jogos de representação são a transformação do comum e popular em algo novo e surpreendente. Os RPGs são atividades em que um grupo de jogadores cooperam para criar uma história oral, escrita ou animada, utilizando a imaginação, esboços, falas, textos, entre outras estratégias de jogo. Cada um dos jogadores representa um papel na história, com exceção de um que assume o papel de narrador, o qual é responsável por descrever o cenário e representar todos os personagens que não são jogados por jogadores. Não existe competição por regra, apenas se isto for definido no cenário do jogo. "A atividade de RPG se apresenta como um sistema de jogo que exige uma participação ativa, democrática, que incentiva o respeito à fala e à ação dos demais jogadores, na construção da narrativa. É uma busca constante para a aquisição do conhecimento, e assim não há perdedores ou vencedores (RODRIGUES, 2004 apud BIFFE, 2018, p. 67).

\section{METODOLOGIA}

A abordagem deste trabalho é qualitativa, uma vez que procura entender e interpretar a performance didática das metodologias ativas de aprendizagem, aplicado na disciplina de Comportamento Humano. De acordo com Lara e Molina (2015), a pesquisa qualitativa é uma expressão a qual possui atividades de investigação que se apresentam de forma específica e possuem características de traços comuns.

Quanto ao objetivo, a pesquisa apresenta um caráter exploratório, pois há um estudo prévio de outras aplicações de metodologias ativas de aprendizagem para formulação e aplicação no curso de Engenharia de Produção. Gehardt e Silveira (2009) afirmam que esse tipo de pesquisa visa com que o pesquisador busque uma maior familiaridade com o problema para que o tema se torne mais explícito e ele consiga construir hipóteses.

Quanto ao método, tem-se uma Pesquisa-ação, já que foi aplicada na sala de aula do curso de Engenharia de Produção, pelos pesquisadores. Conforme Thiollent (1997) apud Neves (2006, p. 11), este é "um tipo de pesquisa social com base empírica que é concebida e realizada em estreita associação com uma ação ou com a resolução de um problema coletivo e no qual os pesquisadores e os participantes representativos da situação ou do problema estão envolvidos de modo cooperativo ou participativo."

\section{MESCLANDO SALA DE AULA INVERTIDA E ROLE PLAY GAME}

As metodologias foram aplicadas na Disciplina de Comportamento Humano do curso de Engenharia de Produção da UTFPR - Campus Londrina. A disciplina é ofertada para alunos do nono período, compreendendo 42 participantes. Optou-se pelo conteúdo: "Gestão de Conflitos", que teve por objetivo desenvolver estratégias de resolução de problemas/conflitos no ambiente organizacional, para promoção do relacionamento interpessoal. A aplicação da metodologia teve duração de 8 horas/aula.

Utilizou-se o formato didático da metodologia Sala de Aula Invertida, as estratégias desse método para a apresentação do conteúdo teórico sobre Gestão de Conflitos, e as atividades préaulas para discussão dos resultados. Para a atividade desenvolvida em sala de aula absorveu a metodologia Role Play Game, com um jogo de representações, promovendo a prática simulada. 


\subsection{Pré-aula (Sala de Aula Invertida) - 2horas/aula}

Para dar início ao conteúdo Gestão de Conflitos, o professor enviou, através da Plataforma Moodle, uma videoaula, de aproximadamente 30 minutos. Nesta videoaula apresentou conceitos de Gestão de Conflitos, estratégias de resolução de problemas e a importância do relacionamento interpessoal no ambiente de trabalho. Após, apresentou um estudo de caso para representar o conteúdo. Os alunos assistiram a videoaula como preparação para a sala de aula. $\mathrm{Na}$ aula, o professor retomou os pontos principais e abriu para dúvidas e questionamentos dos alunos, reforçando o conteúdo proposto.

\subsection{Aula (Role Play Game) - 4horas/aula}

A planejamento e a execução da aula através do RPG, deu-se da seguinte forma:

- Etapa 1: a turma foi dividida em grupos de 6 alunos, totalizando 7 grupos;

- Etapa 2: em seguida, o professor distribuiu fichas com as primeiras orientações sobre RPG, explicando sobre a didática do jogo;

- Etapa 3: com tudo em mãos e mente, cada grupo elegeu um mediador, que narrou todo o cenário do jogo, com a situação-problema. A simulação tratou de um problema sobre a rotulagem de garrafas em uma fábrica de embalagem, apresentando 5 personagens: 1 líder e 4 chefes dos departamentos de qualidade, PCP, Extrusão e Rotulação. Cada chefe tem papéis pré-definidos nas regras do jogo, com características comportamentais e de personalidades para interpretação;

- Etapa 4: cada participante recebeu as características dos personagens para escolha de qual gostaria de interpretar na performance do jogo. Vale mencionar que cada grupo tinha um líder diferente, com estilos de liderança distintos (autoritário, autocrático, democrático, situacional e liberal), provocando caminhos diversos nas tomadas de decisões;

- Etapa 5: definidos os papeis, o jogo iniciou. O grupo teve um tempo determinado para o desenvolvimento da situação-problema apresentada, a partir dos comportamentos definidos nos papeis pré-estabelecidos. O professor, juntamente como mediador, solucionou todos os problemas e dúvidas dos participantes durante o jogo;

- Etapa 6: após a resolução do problema, todos os grupos postaram os relatos dos resultados numa plataforma online, para apresentação e discussão em sala de aula.

Pode-se observar a interação entre os participantes durante a resolução do problema, bem como a forma com que sugeriram e apoiaram as ideias para a solução do problema. A inserção de conhecimentos de outras áreas da Engenharia auxiliou no enriquecimento das ideias de soluções apresentadas. Entretanto, o maior objetivo era avaliar o comportamento dos alunos através das "Estratégias de Liderança", ensinando-os as dificuldades relacionadas ao tema, inserindo-os em uma situação muito próxima da vida real.

\subsection{Pós-aula (Sala de Aula Invertida) - 2horas/aula}

A avaliação se deu através da observação e participação dos alunos no RPG, o professor e o mediador garantiram o engajamento de todos os participantes, tirando dúvidas e auxiliando na interpretação da situação-problema apresentada, garantindo o engajamento de todos na atividade.

Ao final do jogo, o mediador entrou na Plataforma Moodle e preencheu os resultados das discussões do grupo, que posteriormente foi apresentado numa plenária, pelo professor. Após isso, foi aberta para que a turma pudesse compartilhar entre si esses resultados, dada a diversidade do comportamento do líder, uma vez que a construção das personagens-lideres 
apresentaram características diferentes; portanto, comportamentos distintos na resolução de problemas, apresentando uma gama de possibilidades.

Após o compartilhamento, o professor mediou uma discussão elencando os pontos positivos e negativos no comportamento e tomada de decisão do líder, e como isso interfere nos resultados, ou até mesmo promove conflitos, no que concerne o gerenciamento de equipes para resolução de problemas.

\subsection{Percepção dos alunos quanto às metodologias aplicadas}

Os alunos se demonstraram receptivos quanto às metodologias aplicadas, e ao final, foi enviado pela Plataforma Google Forms uma pesquisa de satisfação, utilizando como parâmetros a Escala Likert de 1 a 5: sendo 1 - Insatisfeito, 2 - Parcialmente Insatisfeito, 3 Satisfeito, 4 - Parcialmente Satisfeito, 5 - Totalmente Satisfeito. Foram avaliadas as seguintes categorias:

- Quanto à videoaula, se o aluno assistiu a videoaula sem nenhum problema e se teve compromisso de assistir antes da aula.

- Quanto à participação e envolvimento do aluno durante a aula, se a metodologia promoveu um elo dinâmico de aprendizado;

- Quanto ao desenvolvimento do RPG, se houve dificuldade em desenvolver a performance perante a equipe;

- Quanto à correlação teoria-prática, se o conteúdo teórico desenvolvido na aula expositiva pôde ser aplicado na prática;

- Quanto ao conhecimento adquirido, se houve internalização quanto à Gestão de Conflitos.

Os resultados da pesquisa encontram-se na Figura 2. Observa-se que 26\% dos alunos apresentaram dificuldades e/ou impedimentos para assistir a videoaula, que acaba prejudicando todo o processo de aprendizado. Além das possíveis dificuldades de acesso às tecnologias digitais, esses impedimentos podem estar relacionados à sobrecarga dos alunos, tendo em vista que geralmente eles cursam várias disciplinas no mesmo período e o uso das metodologias ativas acaba exigindo ainda mais dedicação às atividades da disciplina. Verifica-se que $81 \%$ dos participantes compreenderam a metodologia como dinâmica, ou seja, houve um alto índice de participação e envolvimento nas atividades propostas, prevalecendo a organicidade do processo de ensino-aprendizado.

Figura 2 - Pesquisa de Satisfação dos Participantes

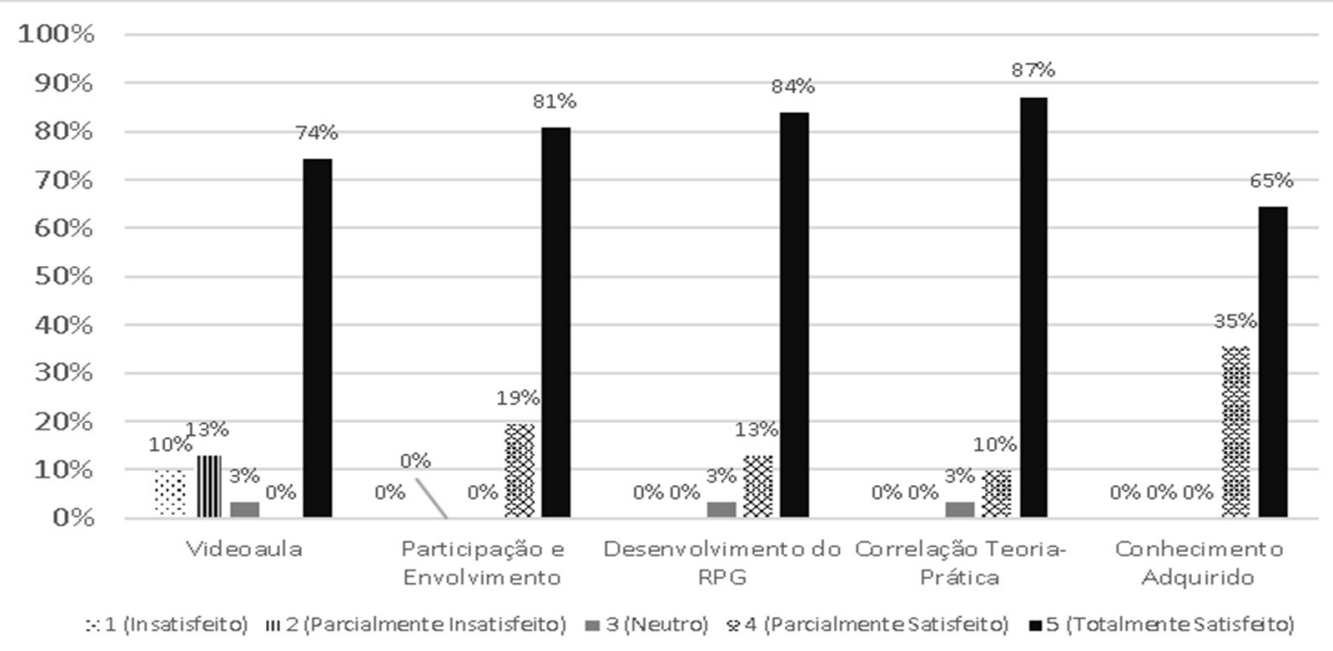

Fonte: Dos autores, 2020. 
Quanto à construção do processo de aprendizagem, 84\% dos participantes conseguiram relacionar o conteúdo teórico às aplicações práticas do jogo RPG, assimilando o conhecimento sobre Gestão de Conflitos, cumprindo com o objetivo do conteúdo ministrado. Por outro lado, $87 \%$ conseguiram, de forma totalmente satisfeita, realizar a relação Teórico-Prática de um modo geral, concebendo o resultado final onde todos os alunos apresentaram um grau parcial ou totalmente satisfeito com o conhecimento adquirido através da metodologia utilizada.

\section{CONSIDERAÇÕES FINAIS}

A atualidade traz uma grande necessidade por parte das universidades de inovar sua forma de ensino, para que o aluno possa, assim, ter uma maior participação ativa em sala de aula e, dessa forma, prender mais seu interesse, além de, também, ser avaliado de forma mais justa.

Cabe, assim, aos docentes inovarem suas atividades em sala para que o aluno tenha essa maior participação, compreensão, bem como desenvolver maior autonomia e segurança, a partir de simulações, nas aplicações práticas, seja num estágio ou trabalho formal.

Ao inserir o aluno em uma situação problema da vida real, facilita-se a assimilação do aprendizado. Assim como, mesclar as metodologias facilitou para preencher as lacunas. Cada método atuou com um objetivo específico sobre o conteúdo Gestão de Conflitos, para garantir a excelência no objetivo geral.

Dessa forma, conclui-se que as metodologias ativas são uma forma diferenciada, participativa e dinâmica de aplicar o conteúdo aos alunos e desenvolver o aprendizado/conhecimento. Além disso, ela também proporciona a troca de ideias, gerando um ambiente colaborativo, que o formato padrão/tradicional de sala de aula não possibilitaria.

Por fim, pode-se concluir que existem ainda muitas alterações a serem feitas nos currículos dos cursos de engenharia, tendo em vista que estes foram formatados para o método tradicional de sala de aula. Assim, seriam necessários estudos que viabilizassem um novo currículo que contemple as metodologias ativas e principalmente a participação ativa do aluno.

\section{REFERÊNCIAS}

AHLERT, Edson Moacir; WILDNER, Maria Claudete Schorr; PADILHA, Teresinha Aparecida Faccio. Metodologias Ativas de Ensino e Aprendizagem. Anais do II Seminário de Educação profissional, 11 e 12 de maio de 2017. Disponível em https://www.univates.br/editora-univates/media/publicacoes/215/pdf_215.pdf

BARBOSA, Paulo Roberto Pereira; ARAÚJO FILHA, Erudina Negreiros de; MIRANDA, Regina Santos de Oliveira; ZANARDI, Sunamita Severino Vilela. Metodologias Ativas no Processo de Aprendizagem Significativa. Revista Olhar Científico. V.04 n.1. Jan/Jul 2018. Disponível em http://www.olharcientifico.kinghost.net/index.php/olhar/article/view/128

BIFFE, Liliane Balaya Martinez. Role Playing Game: Estratégia Didática no Ensino de Gestão em Saúde do Curso Técnico em Segurança do Trabalho. Dissertação de Mestrado. Universidade de Araraquara. Araraquara, 2018. Disponível em https://m.uniara.com.br/arquivos/file/ppg/processos-ensino-gestao-inovacao/producaointelectual/dissertacoes/2018/liliane-blaya-martinez-biffe.pdf 
CABALERO, Sueli da Silva Xavier; MATT, Alfredo Eurico Rodrigues. O Jogo RPG Digital e a Educação: Possibilidade de Aplicação no Ensino Presencial e na EAD. UNEB. 2007. Disponível em http://www.abed.org.br/congresso2007/tc/524200732253PM.pdf

CAVALCANTI, Eduardo Luiz Dias; Soares, Marlon Herbert F. Barbosa. O uso do jogo de roles (roleplaying game) como estratégia de discussão e avaliação do conhecimento químico. Revista Electrónica de Enseñanza de lãs Ciencias. V.8. N.1. 2009. Disponível em http://reec.uvigo.es/volumenes/volumen8/ART14_Vol8_N1.pdf

DE PAULA, Silvio Luiz; DE ALBUQUERQUE, Mariana Cavalcanti Falcão; GRANJA, Brunna Carvalho Almeida; Santos, Claudinete de Fátima Silva Oliveira. Metodologias Ativas: Uma Ação Colaborativa para a Formação de Multiplicadores. Rebecin- Revista Brasileira de Educação em Ciência e Informação. V. $52^{\circ}$ Semestre de 2018. Disponível https://seer.ufs.br/index.php/conci/article/view/10268

ECKERT, Maico Adriano. Metodologias ativas de ensino e aprendizagem. Anais do II Seminário de Educação Profissional. 11 e 12 de maio de 2017. Disponível em https://www.univates.br/editora-univates/media/publicacoes/215/pdf_215.pdf

GEHARDT, Tatiana Engel; SILVEIRA, Denise Tolfo. Métodos de Pesquisa. UFRGS EAD. 2009. Disponível em: http://www.ufrgs.br/cursopgdr/downloadsSerie/derad005.pdf

LARA, Angela Mara de Barros; MOLINA, Adão Aparecido. Capítulo 5: Pesquisa Qualitativa: Apontamentos, Conceitos e Tipologia. 2015. Disponível em: https://gepeto.paginas.ufsc.br/files/2015/03/capitulo-angela.pdf

LEAL, Edvalda Araújo; MIRANDA; Gilberto José; CASA NOVA, Silva Pereira de Castro. Revolucionando a sala de aula: como envolver o estudante aplicando as técnicas de metodologias ativas de aprendizagem. São Paulo, Atlas, 2019.

NEVES, Vanessa Ferraz Almeida. Pesquisa-ação e etnografia: caminhos cruzados. Pesquisas e Práticas Psico Sociais, v1, n1, São João del Rei, jun 2006.

QUIRINO, Maria José da Silva de Oliveira. O Roleplaying Game (RPG) como Estratégia Didática Lúdica: A Qualidade Do Ensino De Ciências. Dissertação de mestrado. IFRJ Nilópolis. 2013. Disponível em http://revistas.unifoa.edu.br/index.php/cadernos/article/view/2245

REZENDE, Márcia; COELHO, Christiano Peres. A Utilização do Role-Playing Game (RPG) no Ensino de Biologia como Ferramenta de Aprendizagem Investigativo/Cooperativa. XXV CONADE- Congresso Nacional da Educação, 2013. Disponível em https://www.academia.edu/3500258/A_Utiliza\%C3\%A7\%C3\%A3o_do_RolePlaying_Game_RPG_no_Ensino_de_Biologia_como_Ferramenta_de_Aprendizagem_Investi gativo_Cooperativa

SCHMIT, Wagner Luiz. RPG e educação: alguns apontamentos teóricos. Dissertação de Mestrado. Universidade Estadual de Londrina, 2008. Disponível em 
"Os desafios para formar hoje o engenheiro do amanhã"

http://www.uel.br/pos/mestredu/images/stories/downloads/dissertacoes/2008/2008\%20-

\%20SCHMIT,\%20Wagner\%20Luiz.pdf

SILVA, Paulo Henrique de Sousa. O Role-Playing Game (RPG) como Ferramenta para o ensino de Física. Dissertação de mestrado. UFRJ. 2013. Disponível em

https://www.if.ufrj.br/ pef/producao_academica/dissertacoes/2016_Paulo_Silva/dissertacao_ Paulo_Silva.pdf

MATOS, Vinícius Costa. Sala de Aula Invertida: Uma Proposta de Ensino e Aprendizagem em Matemática. Dissertação de mestrado, UNB, Brasília, 2018.

RODRIGUES, Carolina Stancati; SPINASSE, Jéssica Fernanda; VOSGERAU, Dilmeire Sant'Anna Ramos. Sala de Aula Invertida - Uma Revisão Sistemática. XII EDUCERE. PUCPR, 2015.

YOSHIZAWA, Erica; PACHECO, Camila; CAMAS, Nuria Pons Vilardell. Sala de Aula Invertida (Flipped Classroom) - Desafios da Formação de Professores para as Demandas Possibilitadas Pelas TIC. XII EDUCERE. 2017. Curitiba- PR

\section{ACTIVE LEARNING METHODOLOGIES AS COLLABORATIVE PRACTICE: MIXING FLIPPED CLASSROOM AND RPG IN THE PRODUCTION ENGINEERING COURSE}

Abstract: The educational scenario brings a long trajectory of teaching based on the transmission of unilateral knowledge, mistakenly centered on the teacher, and the student appeared as a mere passive agent in the learning process, resulting in a high rate of dropout and failure. In the search for teacher updating, active methodologies emerge, which seek to direct teaching strategies towards an active and critical participation of the student, stimulating logical reasoning and an efficient relationship between theory and practice, combined with technological supports. Under this scope, the objective of this article will be to analyze the application of active methodologies, mixing Flipped Classroom and Role Play Game, in the Human Behavior discipline of the Production Engineering course at UTFPR-Campus Londrina, in order to highlight the efficiency of the methods, as well as examining the feasibility of integrating various methodologies in the approach to a content. For this, the method of action research, of an exploratory nature, was used. The results of this research made it possible to conclude that these models allow interaction and motivation of students in the classroom, reaching 100\% satisfaction in the acquisition of knowledge, as well as $81 \%$ of students' through participation and involvement, that is, when mixing two methodologies minimized the learning gaps foreseen by the heterogeneity of the classroom.

Keywords: Active Methodology. Flipped Classroom. Role Play Game. Production Engineering. 